\title{
LES LASERS THULIUM À 2300 NM : AVANCEES ET PERSPECTIVES
}

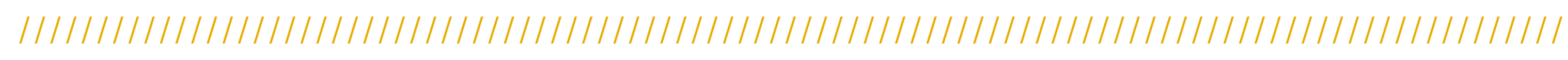

Lauren GUILLEMOT¹, Pavel LOIKO¹, Alain BRAUD, Thomas GODIN², Ammar HIDEUR², Patrice CAMY1

${ }^{1}$ CIMAP UMR 6252 CEA, CNRS, ENSICAEN, Université de Caen, 14050 Caen, France

${ }^{2}$ CORIA UMR 6614, CNRS, INSA, Université de Rouen, 76801 Saint Etienne du Rouvray, France

*hideur@coria.fr

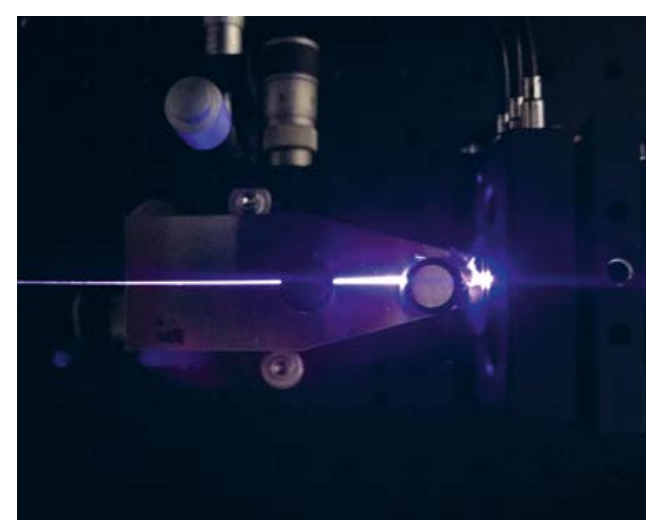

\begin{abstract}
Cet article dresse un état des lieux des dernières avancées dans le domaine des lasers dopés aux ions thulium émettant dans le proche infrarouge autour de $2.3 \mu \mathrm{m}$. Il présente les verrous liés à l'oscillation laser de l'ion thulium sur la transition ${ }^{3} \mathrm{H}_{4} \rightarrow{ }^{3} \mathrm{H}_{5}$ et les solutions prometteuses envisagées pour les contourner en s'appuyant notamment sur un mécanisme de pompage par upconversion particulièrement efficace dans certains matériaux.
\end{abstract}

https://doi.org/10.1051/photon/202110935

Article publié en accès libre sous les conditions définies par la licence Creative Commons Attribution License CC-BY (https://creativecommons.org/licenses/by/4.0), qui autorise sans restrictions l'utilisation, la diffusion, et la reproduction sur quelque support que ce soit, sous réserve de citation correcte de la publication originale.

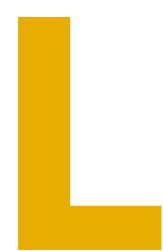

es lasers émettant dans l'infrarouge moyen autour de $2 \mu \mathrm{m}$ sont très attractifs pour plusieurs applications scientifiques, médicales et industrielles. Les applications phares ayant entraîné le développement des lasers émettant dans cette gamme spectrale sont entre autres la lithotripsie (élimination des calculs rénaux), la chirurgie de la prostate ainsi que la détection de polluants atmosphériques, où des lasers holmium sont principalement exploités. Les avancées remarquables réalisées ces dernières années par les lasers à fibre dopée thulium pompés par diodes, qui offrent des rendements opto-électriques élevés et délivrent des puissances de plusieurs kilowatts en régime continu autour de $1.9 \mu \mathrm{m}$, apportent des améliorations considérables à ces applications et ouvrent de nouvelles perspectives pour des applications industrielles, notamment pour le traitement des matériaux mous. En régime d'impulsions ultracourtes, les lasers à fibre à cristaux photoniques dopées au thulium offrent des performances impressionnantes en termes d'énergie extraite à haute cadence. Cependant, ces sources s'appuient sur des procédés de combinaison cohérente complexes et sensibles qui rendent difficile leur déploiement hors laboratoire. L'utilisation de cristaux dopés thulium tels que le YAP dans des architectures conventionnelles d'amplification à impulsion étirée permet de dépasser la barre du millijoule d'énergie en régime sub-picoseconde. Cependant, à ce niveau d'énergie l'absorption par l'eau présente dans l'atmosphère affecte fortement les performances du laser ce qui nécessite de travailler sous atmosphère contrôlée. Une solution très prometteuse pour éviter cette contrainte fondamentale consiste à exploiter la transition à $2.3 \mu \mathrm{m}$ des ions thulium trivalents $\left(\mathrm{Tm}^{3+}\right)$. Cette transition permet en outre une détection du méthane, monoxyde de carbone, formaldéhyde, glucose associée à la possibilité d'une détection à distance puisque le rayonnement à $2.3 \mu \mathrm{m}$ se situe dans la bande $\mathrm{K}$ de transmission de l'atmosphère. Le $\mathrm{Tm}^{3+}$ est tout d'abord bien connu pour son émission laser dans le proche infrarouge à $2 \mu \mathrm{m}$ qui correspond $\bullet \bullet$ 
à la transition ${ }^{3} \mathrm{~F}_{4} \rightarrow{ }^{3} \mathrm{H}_{6}$ (Fig.1). Les matériaux dopés au $\mathrm{Tm}^{3+}$ sont généralement pompés autour de $0.8 \mu \mathrm{m}$ (vers le niveau ${ }^{3} \mathrm{H}_{4}$ ), à l'aide de lasers Titane-Saphir ou de diodes laser AlGaAs de forte puissance. Un processus de relaxation croisée $(\mathrm{CR})$ très efficace entre les ions $\mathrm{Tm}^{3+}$ voisins $\operatorname{Tm}^{1}\left({ }^{3} \mathrm{H}_{4}\right)+\operatorname{Tm}^{2}\left({ }^{3} \mathrm{H}_{6}\right) \rightarrow \operatorname{Tm}^{1}\left({ }^{3} \mathrm{~F}_{4}\right)+\operatorname{Tm}^{2}\left({ }^{3} \mathrm{~F}_{4}\right)$ favorise le peuplement du niveau ${ }^{3} \mathrm{~F}_{4}$. Ainsi, un photon de pompe absorbé génère jusqu'à deux photons lasers, conduisant à une réduction de la charge thermique et à une augmentation du rendement laser dépassant largement la limite de Stokes.

\section{ÉMISSION À 2.3 $\mu \mathrm{m}$ DANS LES MATÉRIAUX DOPÉS THULIUM}

Lémission à $2.3 \mu \mathrm{m}$ qui correspond à la transition ${ }^{3} \mathrm{H}_{4} \rightarrow{ }^{3} \mathrm{H}_{5}$ a été beaucoup moins étudiée. Ses performances sont en effet, à priori, affectées par les relaxations multiphonons qui réduisent la durée de vie du niveau émetteur (en particulier dans les oxydes) et surtout par le processus de CR évoqué précédemment. Chacun de ces mécanismes contribue au dépeuplement du niveau haut de cette transition laser $\left({ }^{3} \mathrm{H}_{4}\right)$, et entraîne une augmentation du seuil laser. La relaxation non-radiative dépend fortement de la matrice hôte et de l'énergie des phonons. Ainsi, les matériaux à faibles énergies de phonons tels que les fluorures $\left(\mathrm{LiYF}_{4}\right.$ et $\mathrm{KY}_{3} \mathrm{~F}_{10}$ ) ou les fibres ZBLAN sont $\grave{a}$ priori plus attractifs pour l'émission laser à $2.3 \mu \mathrm{m}$. En effet, la durée de vie du niveau métastable ${ }^{3} \mathrm{H}_{4}$ est d'environ

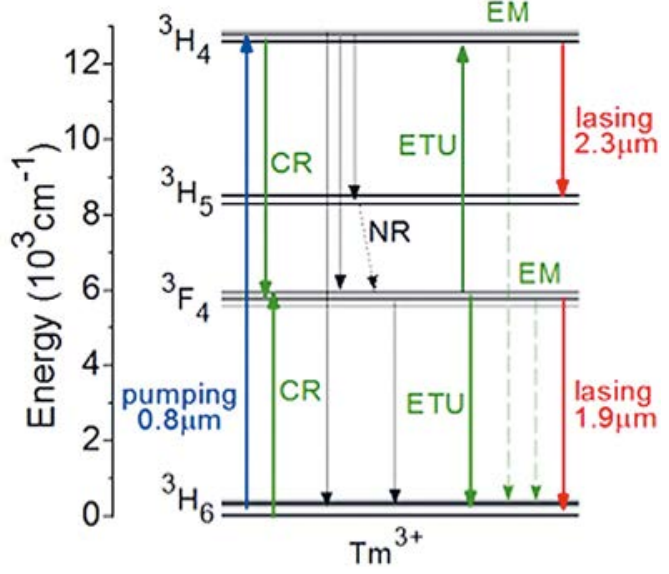

Figure 1. Schéma simplifié des niveaux d'énergie des ions $\mathrm{Tm}^{3+}$ dans $\mathrm{LiYF}_{4}$ : flèches bleues et rouges - transitions de la pompe et du laser, respectivement, flèches noires pleines - relaxation radiative $(\mathrm{R})$, flèche noire en pointillés - relaxation non radiative (NR), flèches vertes - relaxation croisée (CR), upconversion par transfert d'énergie (ETU), migration d'énergie (EM).

$2.3 \mathrm{~ms}$ dans la matrice $\mathrm{LiYF}_{4}$ contre $0.79 \mathrm{~ms}$ dans le $\mathrm{YAG}\left(\mathrm{Y}_{3} \mathrm{Al}_{5} \mathrm{O}_{12}\right)$ à très faible concentration en ions $\mathrm{Tm}^{3+}$. $\mathrm{La}$ relaxation croisée entre ions $\mathrm{Tm}^{3+}$, qui augmente rapidement avec le dopage, a également pour conséquence de fortement réduire la durée de vie du niveau ${ }^{3} \mathrm{H}_{4}$ (voir Fig. 2). Par conséquent, les travaux autour de la transition à $2.3 \mu \mathrm{m}$ du thulium se sont longtemps limités aux matrices vitreuses fluorées faiblement dopées $\mathrm{Tm}^{3+}$ donnant lieu à des rendements faibles insuffisants pour envisager des applications laser.
Des travaux récents montrent que la situation n'est pas aussi désespérée, loin s'en faut. Une meilleure compréhension des processus de transfert d'énergie dans les matériaux dopés $\mathrm{Tm}^{3+}$ permet de favoriser très sensiblement le peuplement du niveau émetteur de la transition à $2.3 \mu \mathrm{m}$. En effet, il a été démontré que le mécanisme de transfert d'énergie par upconversion (ETU) (voir Fig. 1) contribue au repeuplement rapide du niveau ${ }^{3} \mathrm{H}_{4}$ permettant l'utilisation de cristaux fortement dopés thulium pouvant donner lieu à une augmentation significative du rendement quantique qui peut dépasser l'unité pour une émission laser à $2.3 \mu \mathrm{m}$. La mise en évidence de l'impact de ce mécanisme dans un cristal $\mathrm{LiYF}_{4}$ dopé aux ions $\mathrm{Tm}^{3+}$ à 3.5 at.\% a été rapportée en 2019 [1]. Ceci a permis de réaliser un laser quasi-continu délivrant $730 \mathrm{~mW}$ de puissance à $2306 \mathrm{~nm}$ avec un rendement record de $47.3 \%$ par rapport à la puissance pompe absorbée, ce qui correspond à un rendement quantique de 1.27. Le pompage par diode de ce cristal a permis d'obtenir une puissance de sortie de plus de $2 \mathrm{~W}$. L'extension de ces travaux à d'autres cristaux de fluorures $\left(\mathrm{KY}_{3} \mathrm{~F}_{10}\right.$ dopé avec $5 \%$ at. d'ions $\mathrm{Tm}^{3+}$ ), en exploitant le mécanisme de transfert d'énergie par upconversion, a permis de générer une puissance de lordre du watt avec un rendement record de près de $50 \%$ par rapport à la puissance de pompe incidente et un rendement quantique proche de

\begin{tabular}{|c|c|c|c|c|c|c|c|c|}
\hline Matériau & Tm, \% at. & $h v_{p h}, \mathrm{~cm}^{-1}$ & $\sigma_{S E}, 10^{-20} \mathrm{~cm}^{2}$ & $\tau_{30}, m s$ & $\lambda_{p}, n m$ & $P_{\text {out }}, W$ & $\eta, \%$ & $\lambda_{L}, \mu m$ \\
\hline Tm:LiYF 4 & 3.5 & 446 & 0.57 & 2.3 & 780 & 0.73 & 47.3 & 2.31 \\
\hline $\mathrm{Tm}: \mathrm{KY}_{3} \mathrm{~F}_{10}$ & 5 & 495 & 0.34 & 1.9 & 773 & 0.84 & 53.8 & 2.34 \\
\hline $\mathrm{Tm}: \mathrm{BaY}_{2} \mathrm{~F}_{8}$ & 3 & 421 & 0.49 & 1.1 & 779 & 0.1 & 12 & 2.29 \\
\hline $\mathrm{Tm}: \mathrm{YAIO}_{3}$ & 1.8 & 552 & 0.78 & 0.7 & 776 & 0.96 & 61.8 & 2.27 \\
\hline $\mathrm{Tm}: \mathrm{Y}_{3} \mathrm{Al}_{5} \mathrm{O}_{12}$ & 3.2 & 857 & 0.35 & 0.79 & 781 & 1.07 & 46.3 & $2.19-2.32$ \\
\hline $\operatorname{Tm}: \mathrm{KLu}\left(\mathrm{WO}_{4}\right)_{2}$ & 3 & 907 & 1.78 & 0.24 & 793 & 1.12 & 69.2 & $2.22-2.29$ \\
\hline Tm : ZBLAN fiber & $2.5 \% \mathrm{~mol}$. & 550 & 0.21 & 0.22 & 1050 & 1.12 & 37.9 & $2.22-2.29$ \\
\hline
\end{tabular}

$h v_{p h}$, énergie des phonons de la matrice hote; $\sigma_{S E}$, maximum de la section efficace d'émission à $2.3 \mu \mathrm{m}$;

$\tau_{30}$, temps de vie intrinsèque du niveau ${ }^{3} \mathrm{H}_{4} ; \lambda_{\mathrm{p}}$, longueur d'onde de pompe; $\mathrm{P}_{\text {out }}$ puissance de sortie;

$\eta$, rendement laser; $\lambda_{L}$, longueur d'onde laser 

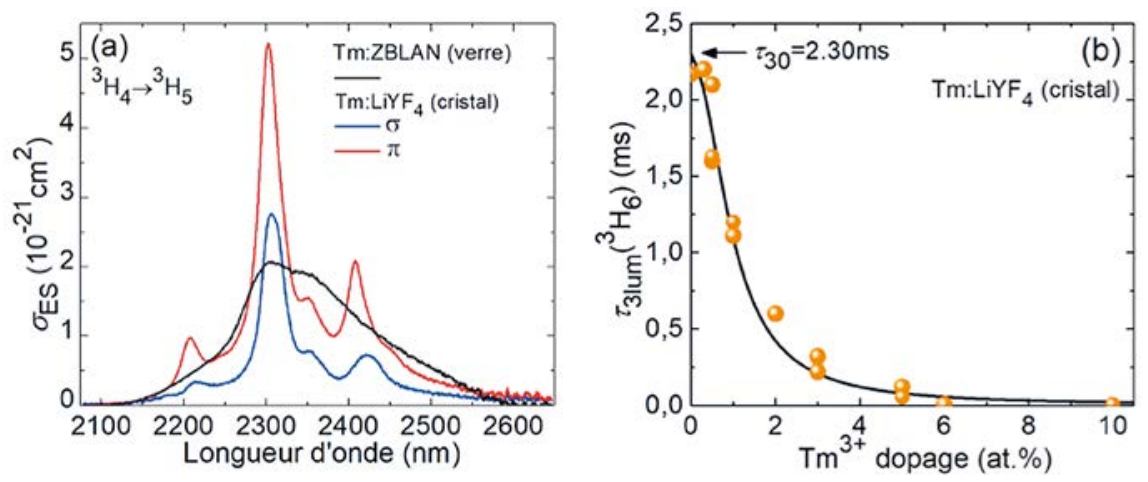

Figure 2. Spectroscopie de la transition ${ }^{3} \mathrm{H}_{4} \rightarrow{ }^{3} \mathrm{H}_{5}$ des ions $\mathrm{Tm}^{3+}$ dans des matrices de fluorures: (a) sections efficaces d'émission stimulée (ES) pour un cristal de fluorure $\left(\mathrm{LiYF}_{4}\right)$ et un verre fluoré (ZBLAN); (b) durée de vie de la luminescence de l'état ${ }^{3} \mathrm{H}_{4}$ dans un cristal Tm : $\mathrm{LiYF}_{4}$ en fonction de la concentration en ions $\mathrm{Tm}^{3+}$. Symboles - données expérimentales provenant de la littérature et courbe - leur ajustement théorique.

deux (voir Fig. 3) [2]. Ces démonstrations ont par ailleurs ouvert la voie à l'étude de matrices d'oxydes possédant pourtant des énergies de phonons plus élevées.

\section{ÉMISSION À $2.3 \mu \mathrm{m}$}

DANS LES OXYDES

Les études spectroscopiques des oxydes dopés $\mathrm{Tm}^{3+}$ tels que le YAP ( $\mathrm{Tm}$ : $\mathrm{YAlO}_{3}$ ) et le YAG ( $\mathrm{Tm}: \mathrm{Y}_{3} \mathrm{Al}_{5} \mathrm{O}_{12}$ ) révèlent que, malgré des énergies de phonons plus élevées que dans les matrices fluorées, la durée de vie du niveau émetteur de la transition à $2.3 \mu \mathrm{m}\left({ }^{3} \mathrm{H}_{4}\right)$ y demeure relativement longue. L'effet de l'ETU, combiné aux grandes sections efficaces de certains de ces matériaux, permet ainsi d'envisager leur exploitation en cavité laser. Les performances obtenues avec des cristaux de Tm:YAG et de YAP [3] montrent qu'il s'agit de candidats sérieux pour la montée en puissance dans le moyen infrarouge en exploitant la transition ${ }^{3} \mathrm{H}_{4} \rightarrow{ }^{3} \mathrm{H}_{5}$ à $2.3 \mu \mathrm{m} \mathrm{du} \mathrm{Tm}^{3+}$. Il a été démontré qu'avec un pompage à $780 \mathrm{~nm}$, il est possible de produire plus de $1 \mathrm{~W}$ de puissance en régime continu avec un rendement de plus de $46 \%$, supérieur à la limite de Stokes, confirmant la contribution du mécanisme de transfert d'énergie par upconversion. Les meilleurs résultats en termes de rendement laser ont été obtenus avec une matrice de tungstate (Tm:Klu $\left.\left(\mathrm{WO}_{4}\right)_{2}\right)$ dopée $\mathrm{Tm}^{3+}$ à hauteur de $3 \%$ qui délivre plus de $1 \mathrm{~W}$ de puissance avec un rendement proche de $70 \%$, ce qui correspond à un rendement quantique proche de 2 . Le tableau en page 36 résume les meilleures performances obtenues à $2.3 \mu \mathrm{m}$ pour les différents matériaux à gain étudiés.

\section{POMPAGE PAR UPCONVERSION}

Avec le pompage conventionnel à $\sim 0.8 \mu \mathrm{m}$, les ions $\mathrm{Tm}^{3+}$ sont directement excités depuis le niveau fondamental ${ }^{3} \mathrm{H}_{6}$ vers le niveau ${ }^{3} \mathrm{H}_{4}$. Toutefois, le niveau ${ }^{3} \mathrm{~F}_{4}$ métastable se trouve également peuplé par le processus de relaxation croisée et par l'émission laser à $2.3 \mu \mathrm{m}$, le niveau ${ }^{3} \mathrm{H}_{5}$ se vidant très rapidement vers le niveau ${ }^{3} \mathrm{~F}_{4}$. Si ce peuplement du niveau ${ }^{3} \mathrm{~F}_{4}$ favorise l'ETU et permet de recycler les ions vers le niveau émetteur, il est aussi favorable à la transition ${ }^{3} \mathrm{~F}_{4} \rightarrow{ }^{3} \mathrm{H}_{6}$ à $1.9 \mu \mathrm{m}$, et entraîne une forte compétition entre les transitions laser à $1.9 \mu \mathrm{m}$ et à $2.3 \mu \mathrm{m}$, en particulier dans les milieux à fort gain tels que les fibres ou les guides d'ondes. Pour contourner cette difficulté, le pompage dit par upconversion constitue une solution attractive. Dans ce schéma de pompage, démontré en 2019 [3], le remplissage du niveau ${ }^{3} \mathrm{H}_{4}$ est obtenu par un processus d'absorption depuis des états excités (ESA). Le mécanisme est amorcé par une très faible absorption de l'état fondamental (GSA) à la longueur d'onde de pompe choisie autour de $1 \mu \mathrm{m}$ ou de $1.45 \mu \mathrm{m}$, relayée ensuite par le processus de relaxation croisée qui remplit efficacementleniveauintermédiaire ${ }^{3} \mathrm{~F}^{4}$, qui $\bullet \bullet$

\section{Laser}

\section{Accordable}

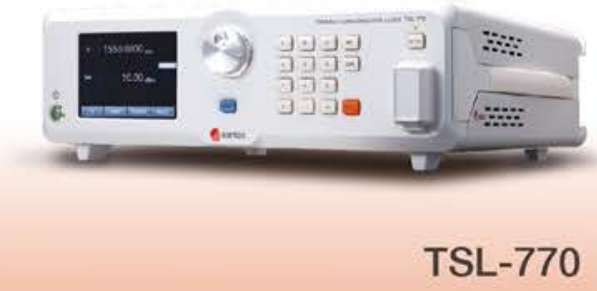

\section{Précis Rapide Stable}

\section{Optiques de pointe}

\section{Télécommunication}

Balayage de 1480 à $1640 \mathrm{~nm}$

Largeur de raie $<60 \mathrm{kHz}$

Vitesse de balayage: $200 \mathrm{~nm} / \mathrm{s}$

Puissance $>+13 \mathrm{dBm}$

Précision $\lambda: 0,3 \mathrm{pm}$

www.santec.com

santec-emea@santec.com 

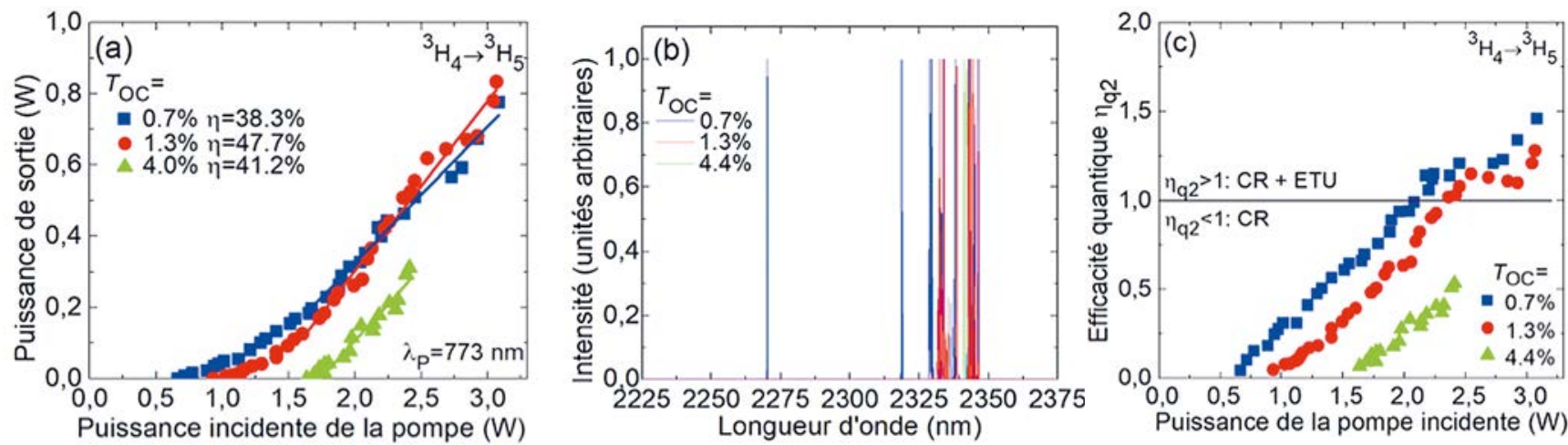

Figure 3. Laser Tm: $\mathrm{KY}_{3} \mathrm{~F}_{10}$ fonctionnant sur la transition ${ }^{3} \mathrm{H}_{4} \rightarrow{ }^{3} \mathrm{H}_{5}$ : (a) dépendance entrée-sortie, $\eta$ - rendement laser; (b) spectre d'émission laser typique; (c) Efficacité quantique de la pompe calculée $\eta_{\mathrm{a} 2}$ impliquant le rôle de l'ETU. L'émission laser est non polarisée, la longueur d'onde de la pompe $\lambda_{p}$ est de $773 \mathrm{~nm}$.

peut être cette fois pompé de façon résonnante à 1.05 ou $1.45 \mu \mathrm{m}$ (voir Fig. 4). Il s'agit d'un mécanisme non linéaire d'avalanche de photons où le niveau ${ }^{3} \mathrm{~F}_{4}$ agit comme un état fondamental effectif préalablement peuplé par un processus d'absorption depuis l'état fondamental assisté par phonons. La mise en œuvre de ce schéma de pompage avec un cristal Tm:LiYF dopé à $3.5 \%$, en exploitant des pompes centrées à 1050 et $1450 \mathrm{~nm}$, montre que le processus de relaxation croisée joue un rôle essentiel. Ce schéma de pompage original a été démontré sur plusieurs cristaux dopés $\mathrm{Tm}^{3+}[4]$ et il s'est montré expérimentalement particulièrement efficace pour le pompage de fibres ZBLAN fortement dopées aux ions $\mathrm{Tm}^{3+}$. En effet, l'exploitation d'un laser à fibre dopée ytterbium émettant autour de $1050 \mathrm{~nm}$ pour le pompage d'une fibre ZBLAN dopée à $2.5 \% \mathrm{~mol}$. a permis de réaliser une source laser opérant en régime quasi-continu délivrant plus de $1 \mathrm{~W}$ de puissance à $2.3 \mu \mathrm{m}$. Ce schéma de pompage est particulièrement prometteur pour les configurations où il est nécessaire de disposer d'une pompe de forte brillance (guides et fibres monomodes), de forte intensité (lasers à blocages de modes basés sur l'effet Kerr) ou qui souffrent d'une compétition marquée avec la transition à $1.9 \mu \mathrm{m}$.

\section{VERS DES SOURCES \\ FEMTOSECONDES À $2.3 \mu \mathrm{m}$}

Les larges bandes d'émission et les rendements élevés des lasers dopés $\mathrm{Tm}^{3+}$ opérant sur la transition à $2.3 \mu \mathrm{m}$ en font des candidats très prometteurs pour la génération et l'amplification d'impulsions ultracourtes dans cette fenêtre spectrale. Les travaux pionniers réalisés ces dernières années ont porté sur les sources de verrouillage de modes à base de cristaux ou de verres fluorés. L'exploitation d'absorbants saturables à semiconducteurs a ainsi permis de réaliser des sources à verrouillage de modes stables mais les temps de relaxation longs de
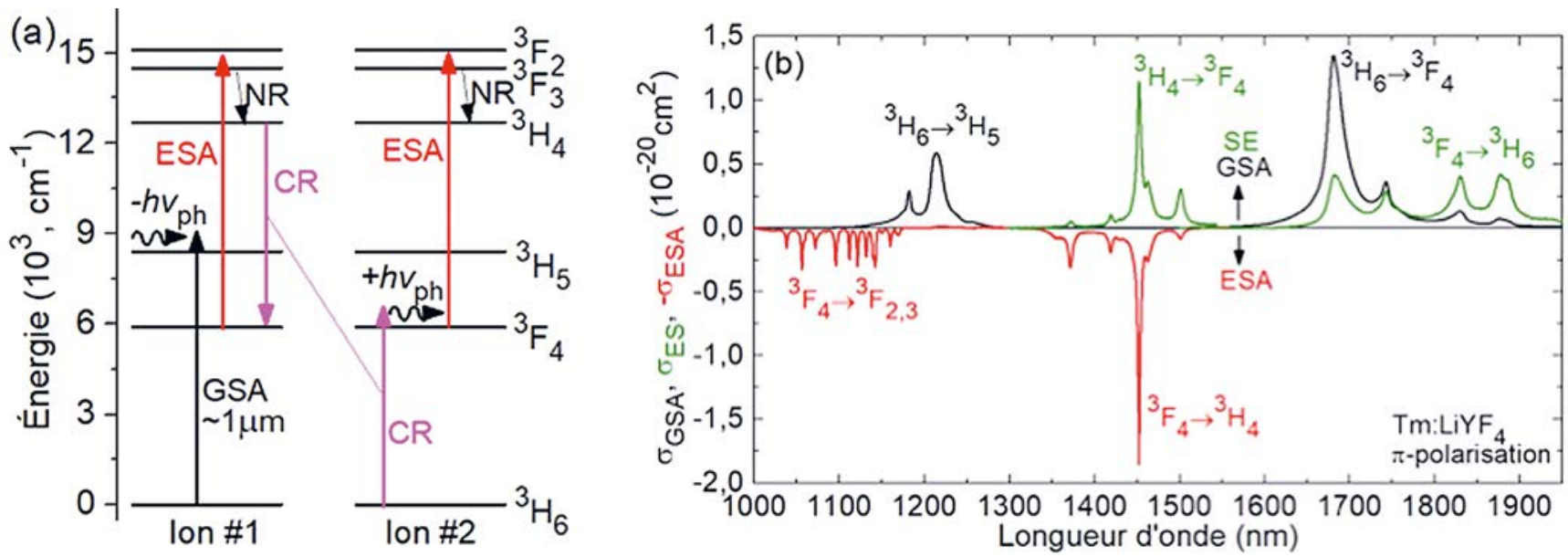

Figure 4. (a) schéma de pompage par « upconversion » d'ions thulium basé sur le mécanisme d'avalanche de photons, GSA et ESA : absorption dans l'état fondamental et dans l'état excité, respectivement; (b) Spectres de GSA et d'ESA dans un cristal Tm:LiYF 4 pour la polarisation $\pi$. 
Il a été démontré que le mécanisme de transfert d'énergie par upconversion (ETU) contribue au repeuplement rapide du niveau ${ }^{3} \mathrm{H}_{4}$ permettant l'utilisation de cristaux fortement dopés thulium pouvant donner lieu à une augmentation significative du rendement quantique qui peut dépasser l'unité pour une émission laser à $2.3 \mu \mathrm{m}$. ces dispositifs limitent à quelques dizaines de picosecondes la durée des impulsions générées. De meilleurs résultats sont obtenus en exploitant le mécanisme de verrouillage de modes par lentille Kerr ou en exploitant des absorbants saturables à base de graphène [5]. En particulier, des sources à base de cristaux Tm: $\mathrm{LiYF}_{4}$ et $\mathrm{Tm}: \mathrm{KY}_{3} \mathrm{~F}_{10}$ exploitant un miroir de graphène ont permis de produire des impulsions sub-picoseconde avec des énergies de quelques nanojoules. La forte stabilité de ces sources opérant à $2.3 \mu \mathrm{m}$, qui ne nécessitent aucun contrôle de l'atmosphère de la cavité, couplée à la possibilité de pomper ces systèmes avec des sources de très hautes puissances et de forte brillance, indiquent que cette nouvelle génération de sources ultrarapides est très prometteuse pour la montée en énergie dans le moyen IR.

\section{CONCLUSION}

Des avancées déterminantes ont été accomplies avec les sources lasers émettant autour de $2.3 \mu \mathrm{m}$ sur la transition ${ }^{3} \mathrm{H}_{4} \rightarrow{ }^{3} \mathrm{H}_{5}$ des ions thulium. Une meilleure maitrise des mécanismes de transfert d'énergie des ions de terres rares dans les matériaux considérés a permis de montrer tout l'intérêt de cette transition encore peu étudiée dans les lasers à solide.
Les travaux récents montrent que les mécanismes de transfert d'énergie par upconversion peuvent être exploités pour compenser les effets de relaxation croisée entre ions $\mathrm{Tm}^{3+}$ et ainsi repeupler le niveau émetteur de la transition à $2.3 \mu \mathrm{m}$. Le principe a été montré sur une large palette de matériaux dopés $\mathrm{Tm}^{3+}$ dans différentes architectures (cristaux massifs, guides d'ondes, fibres optiques) pour réaliser des sources laser efficaces à $2.3 \mu \mathrm{m}$. Un schéma de pompage original à $1.05 \mu \mathrm{m}$, basé sur un effet d'avalanche, a également été démontré. L'extension de ces développements vers les oxydes ayant de très bonnes propriétés thermiques et l'exploitation des bandes larges de certains matériaux ouvrent, de plus, de nouvelles perspectives pour la réalisation de sources lasers de puissance et la génération d'impulsions ultracourtes.

\section{RÉFÉRENCES}

[1] P. Loiko, R. Soulard, L. Guillemot et al., IEEE J. Quantum Electron. 55, 1700212 (2019)

[2] L. Guillemot, P. Loiko, R. Soulard et al., Opt. Express 28, 3451-3463 (2020)

[3] L. Guillemot, P. Loiko, A. Braud et al., Opt. Lett. 44, 5077-5080 (2019)

[4] L. Guillemot, P. Loiko, R. Soulard et al., Opt. Lett. 44, 4071-4074 (2019)

[5] A. Muti, F. Canbaz, M. Tonelli et al., Opt. Lett. 45, 656-659 (2020)

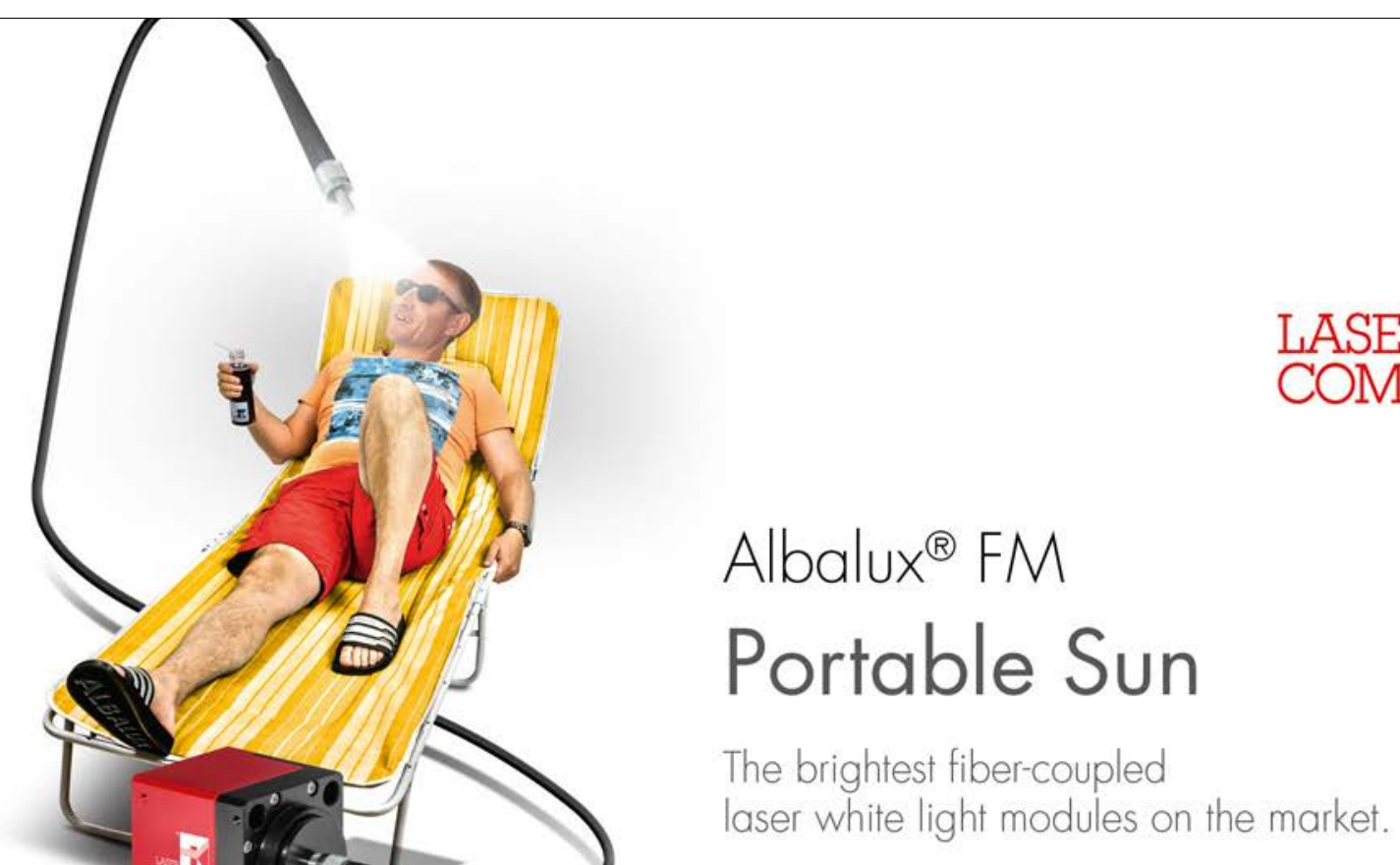

